\title{
Assessment of Longitudinal Reproducibility of Mice LV Function Parameters at 11.7 T Derived from Self-Gated CINE MRI
}

\author{
Zhi Zuo, ${ }^{1,2,3}$ Anne Subgang, ${ }^{3}$ Alireza Abaei, ${ }^{3}$ Wolfgang Rottbauer, ${ }^{1}$ Detlef Stiller, ${ }^{4}$ \\ Genshan $\mathrm{Ma}^{2}$ and Volker Rasche ${ }^{1,3}$ \\ ${ }^{1}$ Department of Internal Medicine II, University Hospital Ulm, Ulm, Germany \\ ${ }^{2}$ Department of Cardiology, Zhongda Hospital, Medical School of Southeast University, Nanjing, China \\ ${ }^{3}$ Core Facility Small Animal MRI, Medical Faculty, Ulm University, Ulm, Germany \\ ${ }^{4}$ In-Vivo Imaging, Drug Development, Boehringer Ingelheim Pharma Gmbh \& Co. KG, Biberach, Germany \\ Correspondence should be addressed to Volker Rasche; volker.rasche@uni-ulm.de
}

Received 10 November 2016; Revised 1 February 2017; Accepted 5 February 2017; Published 22 February 2017

Academic Editor: Cristiana Corsi

Copyright (C) 2017 Zhi Zuo et al. This is an open access article distributed under the Creative Commons Attribution License, which permits unrestricted use, distribution, and reproduction in any medium, provided the original work is properly cited.

\begin{abstract}
The objective of this work was the assessment of the reproducibility of self-gated cardiac MRI in mice at ultra-high-field strength. A group of adult mice $(n=5)$ was followed over 360 days with a standardized MR protocol including reproducible animal position and standardized planning of the scan planes. From the resulting CINE MRI data, global left ventricular (LV) function parameters including end-diastolic volume (EDV), end-systolic volume (ESV), stroke volume (SV), ejection fraction (EF), and left ventricular mass (LVM) were quantified. The reproducibility of the self-gated technique as well as the intragroup variability and longitudinal changes of the investigated parameters was assessed. Self-gated cardiac MRI proved excellent reproducibility of the global LV function parameters, which was in the order of the intragroup variability. Longitudinal assessment did not reveal any significant variations for EDV, ESV, SV, and EF but an expected increase of the LVM with increasing age. In summary, self-gated MRI in combination with a standardized protocol for animal positioning and scan plane planning ensures reproducible assessment of global LV function parameters.
\end{abstract}

\section{Background}

Recent developments in genetic engineering and surgical and pharmacological methods have led to a wide selection of small rodent animal models [1], allowing a better understanding of the underlying mechanisms of cardiovascular diseases and to study new therapeutic interventions. The diagnosis and management of cardiovascular diseases require accurate assessment of cardiac function [2]. The small size of the mouse heart (approximately 1/2000th the mass of a human heart), high heart rates (about 250-650 beats per minute $[\mathrm{bpm}]$, depending on the depth of anesthesia), and high respiratory rates (about 60-160 cycles per minute [cpm]) impose substantial challenges on phenotyping methods.

Echocardiography using high-frequency transducers has proven to be useful in estimating LV function in mice [3]. It is a quick and affordable technique. However, it intrinsically has a relatively low reproducibility since imaging conventionally is $1 \mathrm{D}$ (M-mode) or 2D and so relies on geometric assumptions for volume calculation. Further echocardiography as such is highly dependent on the experience of the user and thus showing high interexaminer variability. Furthermore, shadowing by the sternum and limits on spatial resolution prevent accurate analysis of the ventricles.

Magnetic resonance imaging (MRI) is a noninvasive technique that uses intrinsic tissue contrast and is capable of obtaining volumetric data of the heart and the vascular system. In numerous previous studies, cardiovascular magnetic resonance (CMR) imaging has proven to be an accurate technique and is considered the gold standard for noninvasive assessment of left ventricle (LV) function parameters in humans $[4,5]$. The advantages of CMR are derived from the high soft tissue contrast between blood and myocardium, high spatial and temporal resolution, and the ability to acquire arbitrary slice orientations. On dedicated small animal systems, CMR is able to meet the requirements of the 
TABLE 1: Acquisition parameters of the investigated MR protocol (MS survey: multistack survey; 2/4CH: two/four-chamber; SAX: short axes orientations; $T_{\mathrm{ACQ}}$ : total acquisition time).

\begin{tabular}{|c|c|c|c|c|c|c|}
\hline & MS survey & Semi-2CH & Semi-4CH & SAX & $2 \mathrm{CH}$ & $4 \mathrm{CH}$ \\
\hline$T_{E} / T_{R}[\mathrm{~ms}]$ & $1.25 / 23.5$ & $1.25 / 20$ & $1.25 / 20$ & $1.0 / 5.75$ & $1.0 / 5.75$ & $1.0 / 5.75$ \\
\hline Gated & No & Yes & Yes & Yes & Yes & Yes \\
\hline Phases & NA & 10 & 10 & 20 & 20 & 20 \\
\hline In-plane resolution $[\mu \mathrm{m}]$ & $117 \times 117$ & $117 \times 117$ & $117 \times 117$ & $117 \times 117$ & $117 \times 117$ & $117 \times 117$ \\
\hline Slice thickness $[\mu \mathrm{m}]$ & 1000 & 1000 & 1000 & 500 & 500 & 500 \\
\hline Number of repetitions & NA & 75 & 75 & 200 & 200 & 200 \\
\hline$T_{\mathrm{ACQ}}[\mathrm{s}]$ & 47.185 & 40.4 & 40.4 & 1305.604 & 163.2 & 163.2 \\
\hline
\end{tabular}

small-sized, fast-beating mouse heart and has thus emerged as the most accurate imaging modality currently available for deriving detailed, noninvasive assessments of cardiac structure and function in mice $[6,7]$. With the recent developments of self-gated cardiac imaging [8], data acquisition has been dramatically simplified and cardiac imaging in small rodents is ready to become mainstay for assessment of cardiac function.

Where developmental studies have been done by Wiesmann et al. for assessing the cardiac function and mass between neonatal (day 3) and juvenile (week 16) age after birth [7], reproducibility studies especially of the self-gated techniques are limited. Recent studies include longitudinal assessment of cardiac function and mass at a limited number of time points $[9,10]$, application to rats [11-14], or low fieldstrength applications [15-18]. Further studies include assessment of the intra- and interobserver reproducibility [9], with Vanhoutte et al. applying the simplified self-gated imaging technique. Even though the investigations showed excellent reproducibility of cardiac function and left ventricular mass with rather low inter- and intraobserver variability, studies of the longitudinal reproducibility, which are important for assessment of the sensitivity of CMR for changes of cardiac parameters over time, have not been published, yet.

For assessment of the longitudinal reproducibility of CMR-derived cardiac parameters at $11.7 \mathrm{~T}$, in this study a group of adult mice were followed for 360 days (postnatal day (PND) 91-PND 451) with a standardized imaging protocol for assessment of changes of cardiac parameters over time and the reproducibility of the imaging protocol.

\section{Methods}

2.1. Ethics Approval. Animal experiments were approved by the regional board of Tübingen and conducted according to German law for the welfare of animals and regulations for care and use of laboratory animals. All institutional and national guidelines for the care and use of laboratory animals were followed and approved by the appropriate institutional committees.

2.2. Animals. Female wild type mice (C57/B6, $n=5)$ were included in this study. Before the start of the study, the animals were acclimatized in a temperature-controlled environment for one week. Facility rooms were maintained at constant temperature $\left(23^{\circ} \mathrm{C}\right)$, humidity $(50 \%$ relative humidity), and $12 \mathrm{~h}$ light-dark illumination cycle. Access to food and tap water was ad libitum.

\subsection{CMR Image Acquisition}

2.3.1. MRI Protocol. Imaging was performed on an 11.7 Tesla small animal MRI system (Bruker BioSpec 117/16, Germany). Data were obtained with a dedicated four-element array-coil optimized for imaging the mouse heart (Bruker BioSpec, Ettlingen, Germany). The MR imaging protocol (Figure 1) comprised a multislab survey acquisition in axial, coronal, and sagittal orientation, followed by two longaxis CINE scans in semi-two-chamber (semi-2CH) and semi-four-chamber (semi-4CH) geometry. The semi-2/4CH images were used to plan the subsequent stack of short axes orientations (SAX). The number of short axis slices was adopted to ensure full coverage of the ventricles in enddiastole. Final 2- and 4-chamber orientations were planned on the SAX data. All cine acquisitions were performed using a self-gating approach (IntraGate $\odot$, Bruker Biospin, Ettlingen, Germany). The principle of the self-gating technique is depicted in Figure 2. In short, the self-gating technique is a modified retrospectively gated Fast Low Angle Shot (FLASH) [19] imaging approach. The major modification results from splitting up the acquisition of each $k$-space line into two subsequently performed acquisitions. During the first acquisition a navigator signal without phase encoding is generated, and with the subsequent acquisition a single line in $k$-space is encoded as well known from the conventional FLASH technique. The navigator signal is derived from the magnitude and phase of few initial points of the free induction decay (FID) after low flip-angle excitation. To maximize heartphase related navigator signal alterations, a volume covering almost the complete ventricles is chosen for generation of the navigator signal. To ensure sufficient data for the subsequent multiphase reconstruction, the acquisition of the complete data set is repeated several times (number of repetitions). During retrospective reconstruction, the acquired data is sorted according to the identified cardiac and respiratory phase thus enabling the reconstruction of cardiac- and respiratory-phase resolved CINE data. Excellent myocardium-blood contrast is obtained by sequential acquisition of the slices thus minimizing the repetition time (TR) and hence saturation effects of blood. Detailed parameters of the different sequences are summarized in Table 1. 


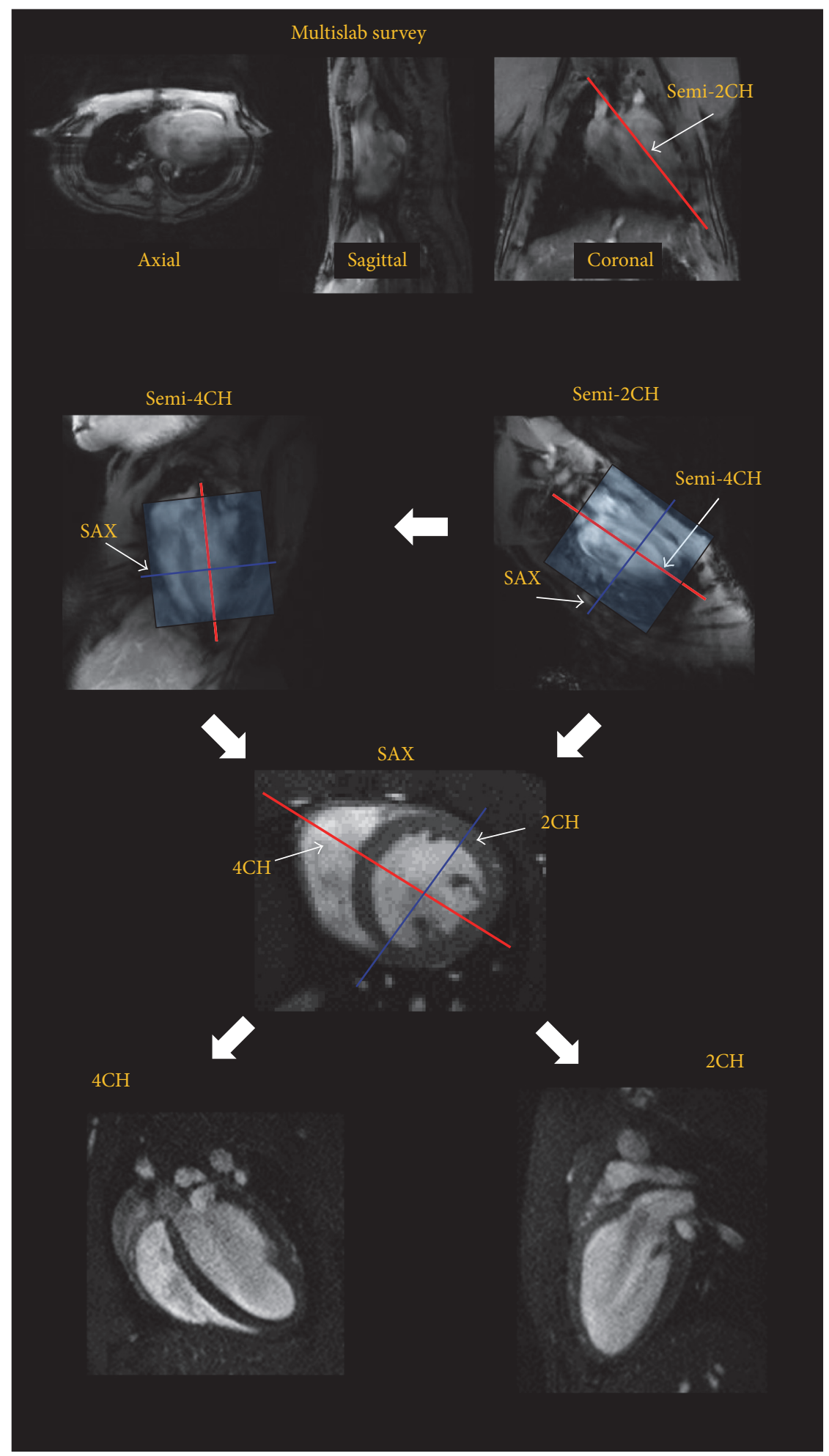

FIGURE 1: Imaging protocol for highly reproducible imaging of cardiac function and anatomy (2/4CH: 2/4-chamber, SAX: short axes orientations).

2.3.2. Imaging Protocol. All animals were scanned under isoflurane anesthesia. For initiation of the anesthesia, 5\% isoflurane concentration in room air was used. For maintaining the anesthesia during the MRI, the isoflurane concentration was adapted to a value resulting in a respiratory frequency between 50 and 70 respiratory cycles per minute. Depending on the animal, isoflurane concentrations between $1 \%$ and $1.5 \%$ were required. During scanning the respiratory rate 


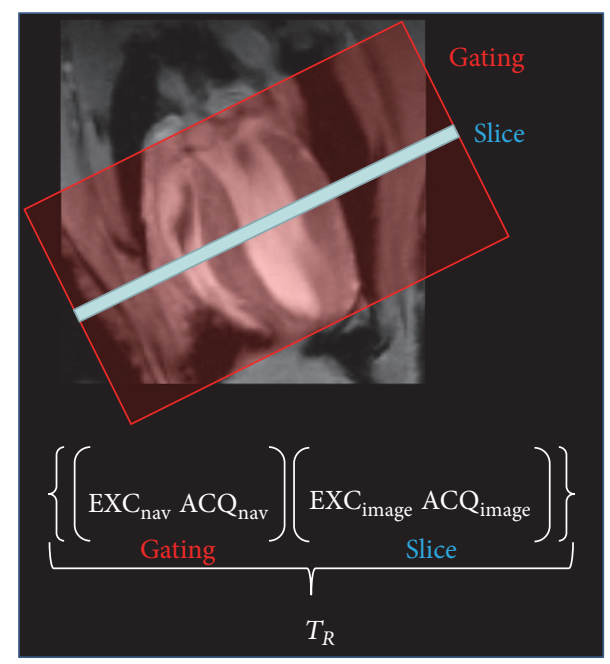

FIgURE 2: Principle of the IntraGate CINE imaging technique.

was monitored and the temperature of the animals was controlled by water heating of the animal bed. The animals were positioned in prone position carefully taking care for straight alignment of the animal with the animal support. The respiratory cushion was placed between the abdominal wall and the animal cradle at the level of the liver region to minimize its impact on the positioning of the animal in the target area.

All animals were followed over 360 days and scanned following the MRI protocol mentioned above at 9 time points on 6 different days (PND 91, PND 93, PND 95, PND 151, PND 211, and PND 451 after birth). On PND 91, PND 93, and PND 95, the entire protocol was performed twice within 2 hours, yielding 9 measurements per animal in total. Between the subsequently performed scans on PND 91, PND 93, and PND 95 , the animals were completely taken out of the scanner and repositioned.

2.4. CMR Image Analysis. All data were carefully analyzed regarding image quality. Data sets with significant artifacts caused by unexpected animal motion in at least a single slice were excluded. In this study no rescanning of corrupted slices was performed to ensure the same imaging protocol in all animals. LV global function parameters were evaluated by tracing epicardial and endocardial borders in end-diastole and end-systole. Contours were manually identified in all short axis images from base to apex. During delineation of the endocardial contour, papillary muscles were included as part of the myocardial wall. The apical slice was identified as the most distal segment that contains LV cavity and myocardium. The basal slice was defined as the most proximal segment located above the tips of the papillary muscles at the chordae tendineae. End-diastolic volume (EDV), end-systolic volume (ESV), and stroke volume (SV) were determined and LV ejection fraction (EF) and end-diastolic left ventricular mass (LVM) derived. The average time for image postprocessing was about $15 \mathrm{~min}$ per animal. All image analyses were done blinded using the freely available software Segment version 1.9.1 (http://segment.heiberg.se) [20].
2.5. Statistical Analysis. The data were used for assessing the (a) reproducibility or interexperiment variability of the proposed imaging approach, (b) the variability of the function parameters within a group, and (c) the longitudinal changes of the function parameters over one year.

Regarding (a) the assessment of the reproducibility of the suggested imaging protocol was performed based on the comparison of the function parameters of the subsequent acquired scans performed on PND 91, PND 93, and PND 95.

The mean and standard deviation were computed for each parameter and each measurement point. Statistical significance of differences between subsequent measurements was assessed applying a two-way ANOVA test with $p$ values of $p \leq 0.05$ being considered significant. The agreement between two measurements was assessed by mean of BlandAltman analysis. Coefficient of variance $(\mathrm{CoV})$ was calculated for assessing the variability within repeated measurements.

Regarding (b) intragroup variability of LV function parameters was assessed for all investigated parameters (EF, EDV, ESV, SV, and LVM) separately for all 9 time points, calculation of the respective $\mathrm{CoV}$. The mean intragroup variability was derived for each parameter as the mean of the individual $\mathrm{CoV}$ of each of the 9 measurements.

Regarding (c) longitudinal variability was assessed over 360 days. ANOVA test was used to investigate the significance of changes over the $360 \mathrm{~d}$ period for all derived LV parameters. For PND 91, PND 93, and PND 95, only the first measurement was included in the ANOVA analysis. CoV of each LV parameter was computed for each mouse.

The $\mathrm{CoV}$ was calculated as the fraction of standard deviation and mean of the respective function values. The reproducibility of the investigated parameters was classified according to the $\mathrm{CoV}$ value to excellent $(\mathrm{CoV}<10 \%)$, very good $(\mathrm{CoV} \geq 10 \%$ and $<15 \%)$, good $(\mathrm{CoV} \geq 15 \%$ and $<20 \%)$, moderate $(\mathrm{CoV} \geq 20 \%$ and $<25 \%)$, acceptable $(\mathrm{CoV} \geq 25 \%$ and $<30 \%)$, and poor $(\mathrm{CoV} \geq 30 \%)$.

ANOVA analysis was performed with SPSS 17.0 (IBM, USA). Bland-Altman test was performed with MedCalc 15.8 (MedCalc Software bvba, Belgium).

\section{Results}

The average total scan time resulted in about 30 minutes per animal. Mean respiratory and heart rates of the animals were $60 \pm 8(\min =47, \max =78) \mathrm{cpm}$ and $398 \pm 68(\min =296, \max$ $=565) \mathrm{bpm}$. All scans could be completed in all animals. In total, 7 out of the 45 image stacks were excluded from further analysis due to at least one motion corrupted slice in the short axes image stack.

Figure 3 shows short axis images acquired at mid-ventricular level along the long axis of the heart for all acquired time points exemplarily for one animal. Even though each of the scans was performed after completely repositioning of the animal and independent scan planning, the images indicate the high reproducibility of the investigated technique.

3.1. Reproducibility of the Imaging Protocol. For all investigated parameters, the mean $\pm \mathrm{SD}$, Bland-Altman analysis, $p$ value, and $\mathrm{CoV}$ resulting from the comparison of the 

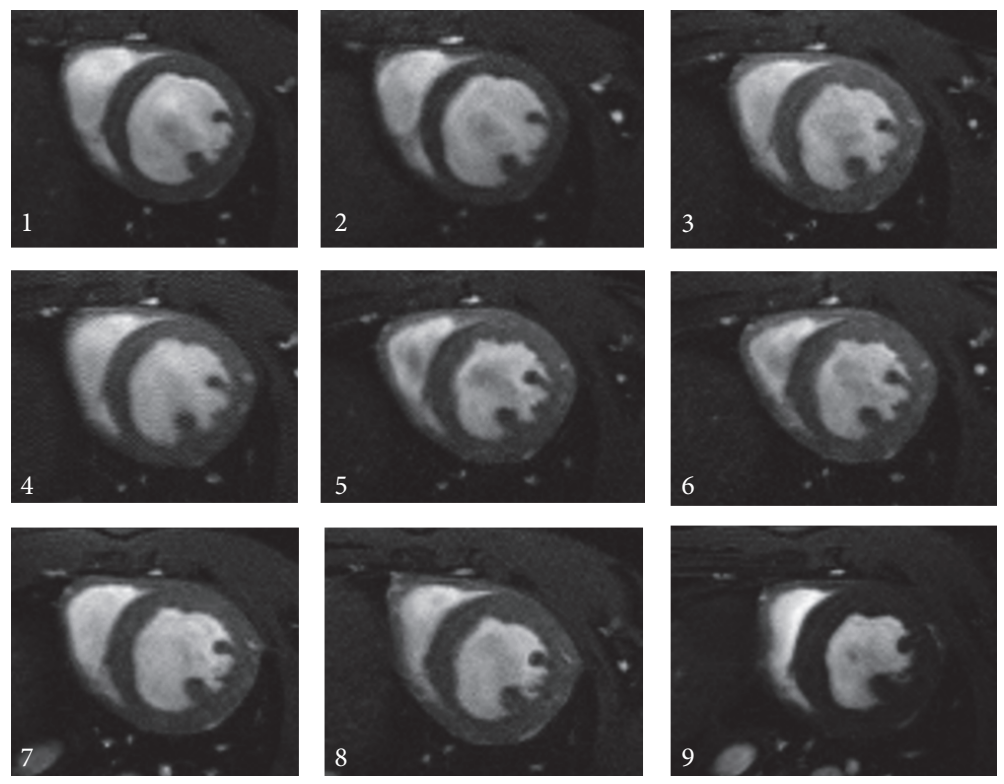

FIGURE 3: End-diastolic CINE magnetic resonance short axis images, acquired at a mid-ventricular level at measurement 1 of day 91 (1), measurement 2 of day 91 (2), measurement 1 of day 93 (3), measurement 2 of day 93 (4), measurement 1 of day 95 (5), and measurement 2 of day 95 (6), day 161 (7), day 211 (8), and day 451 (9) after birth.

TABLE 2: Interexperiment comparison of the reproducibility and variability of cardiac function parameters and left ventricular mass (LVM).

\begin{tabular}{lccccc}
\hline & 1st measurement & 2nd measurement & Bias & 95\% CI & $p$ \\
\hline EF $(\%)$ & $71.17 \pm 4.99$ & $69.83 \pm 6.38$ & $1.33 \pm 5.42$ & $-2.11 \sim 4.77$ & 0.583 \\
EDV $(\mu \mathrm{l})$ & $49.42 \pm 5.82$ & $50.58 \pm 9.03$ & $-1.17 \pm 6.28$ & $-5.16 \sim 2.82$ & 0.644 \\
ESV $(\mu \mathrm{l})$ & $14.42 \pm 3.32$ & $15.42 \pm 4.38$ & $-1.00 \pm 3.72$ & $-3.36 \sim 1.36$ & 0.496 \\
SV $(\mu \mathrm{l})$ & $35.25 \pm 4.29$ & $35.42 \pm 6.73$ & $-0.17 \pm 4.63$ & $-5.16 \sim 2.82$ & 0.935 \\
LVM $(\mathrm{mg})$ & $81.46 \pm 4.95$ & $80.06 \pm 6.19$ & $1.40 \pm 4.99$ & $-1.77 \sim 4.57$ & 0.554 \\
\hline
\end{tabular}

two subsequent measurements are summarized in Table 2. The respective Bland-Altman plots are provided in Figure 4. Between the two subsequent measurements acquired the same day, no significant differences were observed for all investigated parameters $(\mathrm{dEF}=1.33 \%$, dEDV $=-1.17 \mathrm{ml}$, $\mathrm{dESV}=-1.00 \mathrm{ml}$, dSV $=-0.17 \mathrm{ml}$, and $\mathrm{dLVM}=1.40 \mathrm{mg}$ ). For EF and LVM excellent, for EDV and SV very good, and ESV moderate reproducibility was observed.

3.2. Intragroup Variability. The intragroup variability for all measurements and the respective mean values are provided in Table 3. Similar to the reproducibility measurements, intragroup variability was excellent for EF and LVW and very good for EDV and SV, but only moderate for the ESV.

3.3. Longitudinal Variability. The longitudinal outcome for the investigated parameters is shown in Figure 5. From the longitudinal mean values, it becomes quite obvious that the EF is by far the most reliable parameter and EDV, ESV, and SV show more variation. For the LVM a longitudinal increase is observed.

The observations are confirmed by the ANOVA analysis presented in Table 4. Significant changes were observed in LVM only, showing increased values on PND 451. All other investigated parameters do not show significant variation over the one-year term.

$\mathrm{CoV}$ values over all time points for each investigated mouse are shown in Table 5. Longitudinal reproducibility was excellent for the EF, very good for EDV and SV, and moderate for the ESV.

\section{Discussion}

With the increasing use of small animal model in cardiovascular research, there is an increasing demand in noninvasive methods for assessing anatomy and function of the heart and to characterize myocardial tissue. For longitudinal studies, it is of utmost importance to ensure high reproducibility of the applied method to ensure sensitive identification of different outcomes even in rather small group sizes.

Currently, there is no established gold standard for assessment of cardiac function for small animals. Even though echocardiography is frequently applied, an only limited reproducibility for cardiac function parameters has been reported [21]. Factors such as usage of models for final calculation of volumetric data from few two-dimensional slices limit the accuracy and reproducibility of the technique. This is 

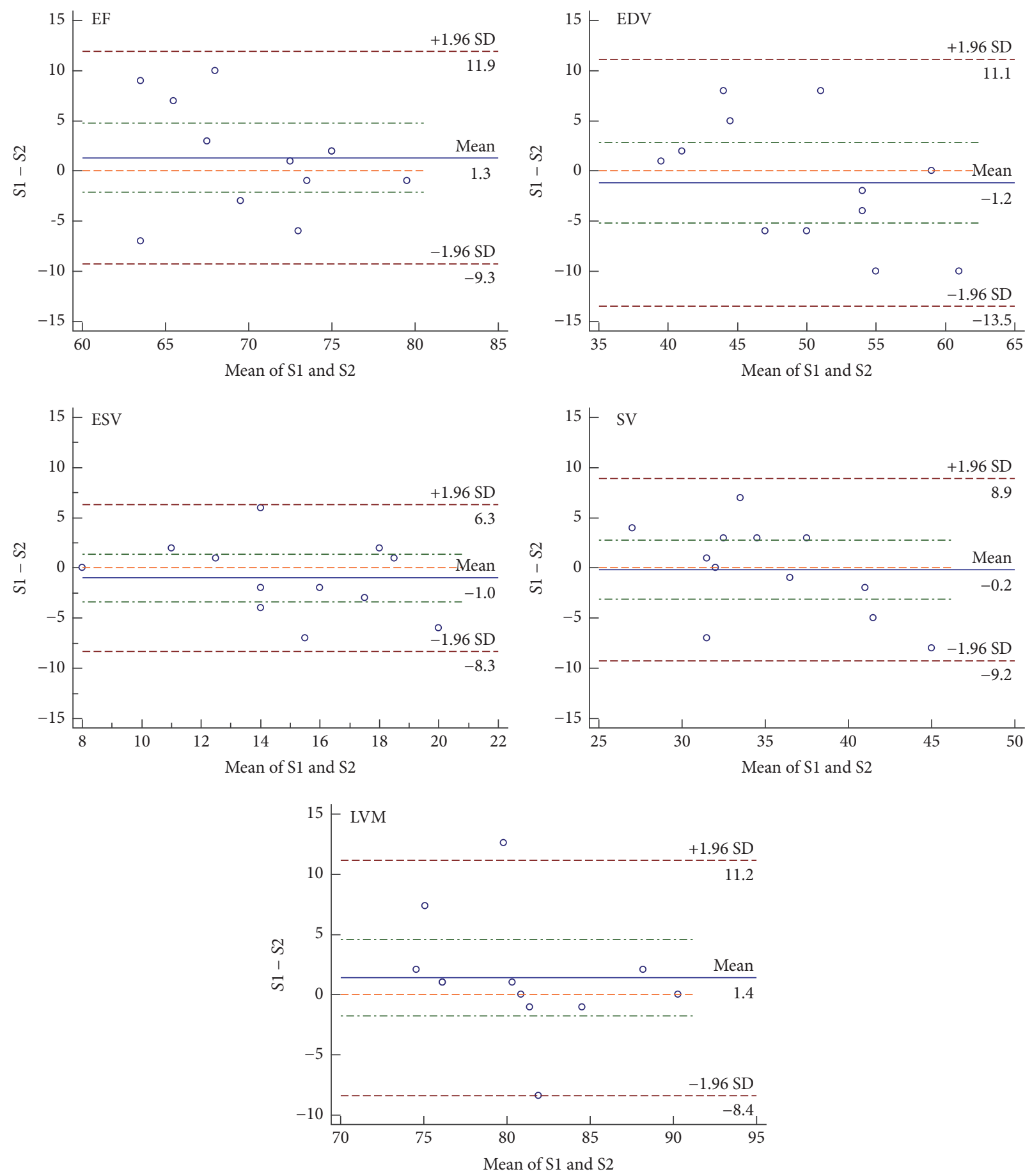

FIGURE 4: Bland-Altman plots of the differences between the subsequent measurements (S1, S2) acquired the same day.

especially obvious in many animal models of cardiovascular disease showing abnormal ventricular geometry or heterogeneously regional cardiac function [22].

With CMR a versatile imaging tool is available, which enables noninvasive assessment of cardiac anatomy and function, and in combination with advanced imaging techniques has the potential to enable myocardial tissue characterization. In direct comparison with high-frequency echocardiography,
MRI appears especially advantageous in animals cardiac pathologies impacting left ventricular geometry of regional function, cases in which echocardiography often lacks accuracy [22]. In particular, the full-volume acquisition possible with MRI was shown to yield improved reproducibility when compared with echocardiography [12]. There is only a small body of literature comparing MRI and echocardiography in the same animal model but there is strong evidence that 


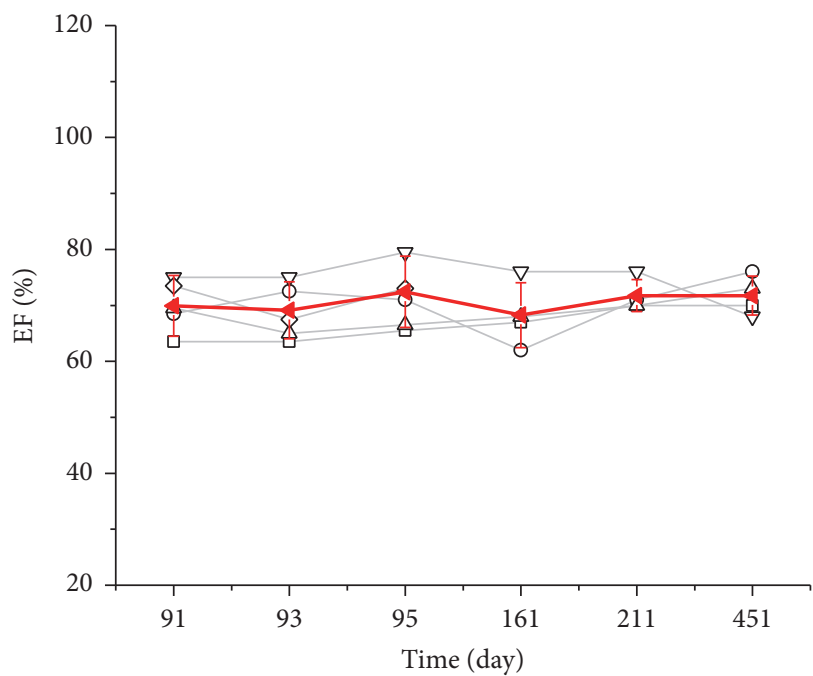

(a)

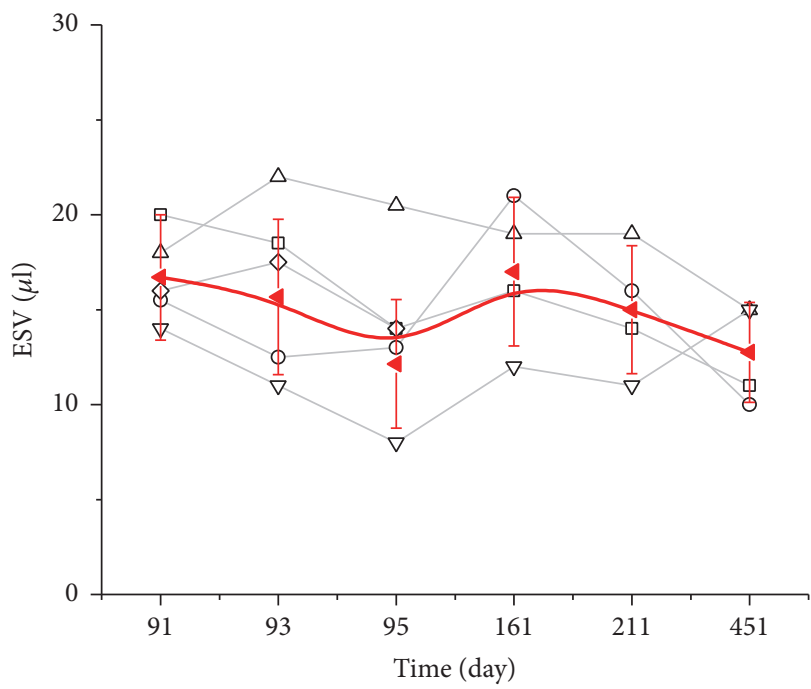

(c)

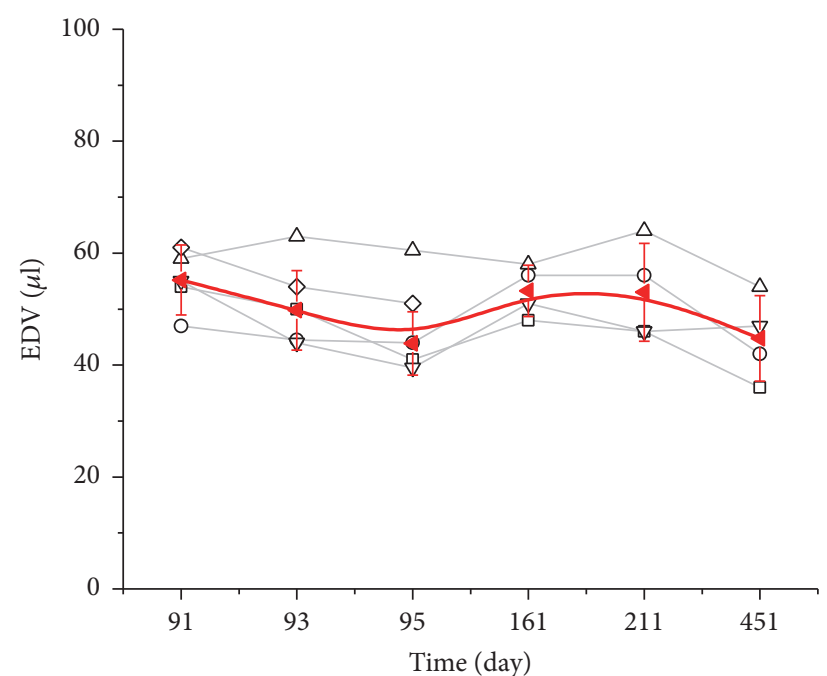

(b)

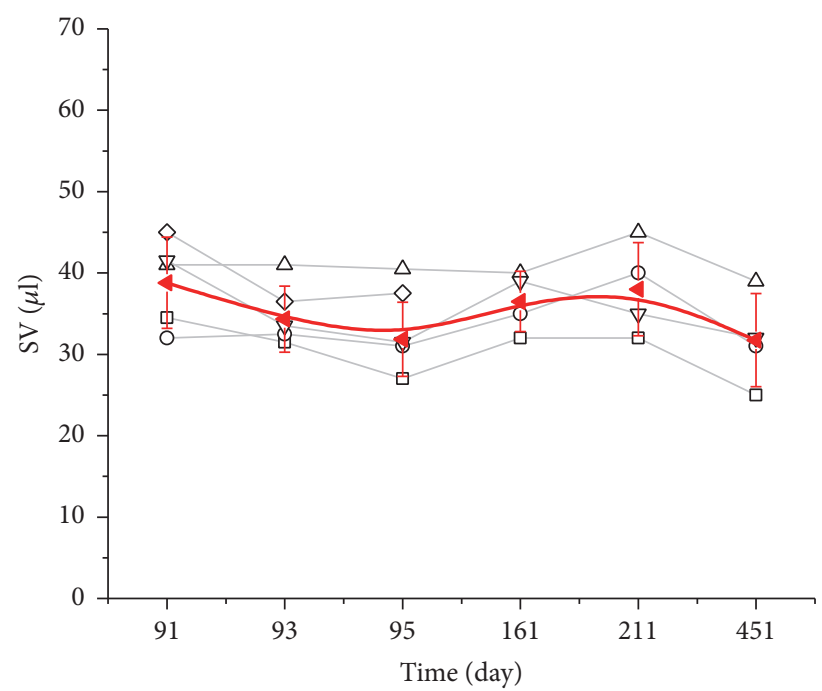

(d)

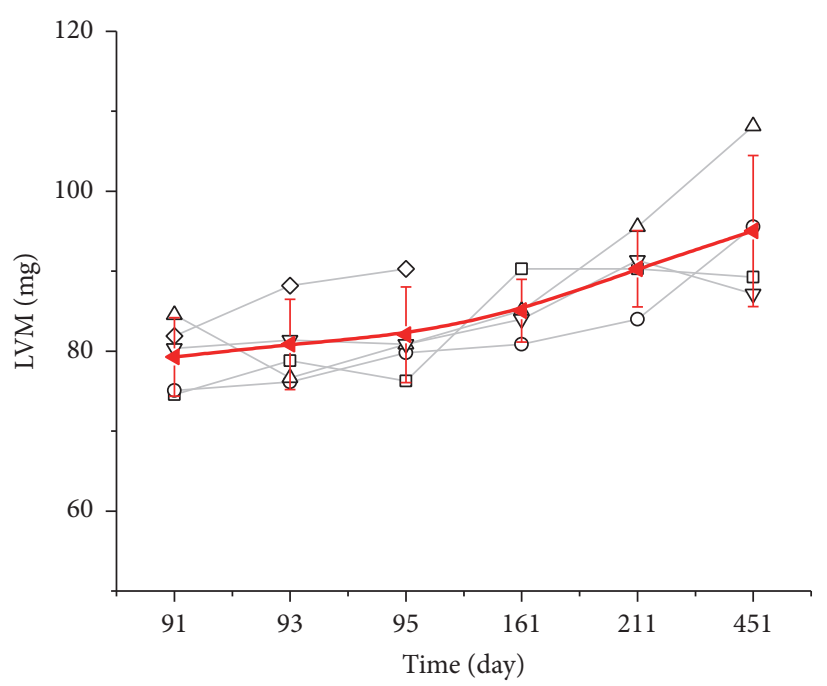

(e)

FIgURE 5: Individual (light) and mean (bold, red) left ventricular function parameters and mass for the six acquisition time points. 
TABLE 3: CoV (\%) of left ventricular function parameters and left ventricular mass (LVM) for all investigated time points and the resulting mean value.

\begin{tabular}{|c|c|c|c|c|c|}
\hline & $\mathrm{CoV}(\mathrm{EF})$ & $\mathrm{CoV}(\mathrm{EDV})$ & $\mathrm{CoV}(\mathrm{ESV})$ & $\mathrm{CoV}(\mathrm{SV})$ & $\mathrm{CoV}(\mathrm{LVM})$ \\
\hline PND 91 1st measurement & 4.90 & 11.01 & 20.55 & 9.70 & 4.03 \\
\hline PND 91 2nd measurement & 10.02 & 10.27 & 15.18 & 18.29 & 8.37 \\
\hline PND 93 1st measurement & 9.25 & 13.24 & 25.36 & 13.54 & 6.90 \\
\hline PND 93 2nd measurement & 5.54 & 16.61 & 30.00 & 9.94 & 7.80 \\
\hline PND 95 1st measurement & 6.33 & 14.81 & 28.91 & 13.28 & 7.18 \\
\hline PND 95 2nd measurement & 13.01 & 10.38 & 30.99 & 17.96 & 9.06 \\
\hline PND 161 & 8.49 & 8.59 & 23.03 & 10.13 & 4.62 \\
\hline PND 211 & 4.00 & 16.45 & 22.44 & 15.04 & 5.29 \\
\hline PND 451 & 4.88 & 17.06 & 20.63 & 18.07 & 9.94 \\
\hline Mean & $7.38 \pm 2.99$ & $13.16 \pm 3.21$ & $24.12 \pm 5.19$ & $13.99 \pm 3.58$ & $7.02 \pm 2.03$ \\
\hline
\end{tabular}

TABLE 4: ANOVA analysis of longitudinal variability of LV parameters. Significant differences were observed only in the left ventricular mass.

\begin{tabular}{lccccccc}
\hline & PND 91 & PND 93 & PND 95 & PND 161 & PND 211 & PND 451 \\
\hline EF $(\%)$ & $71.6 \pm 3.51$ & $68.6 \pm 6.35$ & $72.25 \pm 4.57$ & $68.25 \pm 5.80$ & $71.75 \pm 2.87$ & $71.75 \pm 3.5$ & 0.680 \\
EDV $(\mu \mathrm{l})$ & $52.4 \pm 5.77$ & $51.4 \pm 6.80$ & $45.25 \pm 6.70$ & $53.25 \pm 4.57$ & $53 \pm 8.72$ & $44.75 \pm 7.63$ & 0.274 \\
ESV $(\mu \mathrm{l})$ & $15 \pm 3.08$ & $16.4 \pm 4.16$ & $12.75 \pm 3.69$ & $21 \pm 7.12$ & $20.75 \pm 3.77$ & $18.75 \pm 5.74$ & 0.118 \\
SV $(\mu \mathrm{l})$ & $37.6 \pm 3.65$ & $35.2 \pm 4.76$ & $32.75 \pm 4.35$ & $36.5 \pm 3.70$ & $38 \pm 5.72$ & $31.75 \pm 5.74$ & 0.321 \\
LVM $(\mathrm{mg})$ & $79.38 \pm 3.20$ & $81.9 \pm 5.65$ & $81.9 \pm 5.88$ & $85.05 \pm 3.93$ & $90.3 \pm 4.77$ & $95.03 \pm 9.45$ & 0.006 \\
\hline
\end{tabular}

TABLE 5: CoV (\%) of left ventricular function parameters for each investigated mouse over the 9 time points.

\begin{tabular}{lcccc}
\hline & $\mathrm{CoV}(\mathrm{EF})$ & $\mathrm{CoV}(\mathrm{EDV})$ & $\mathrm{CoV}(\mathrm{ESV})$ & $\mathrm{CoV}(\mathrm{SV})$ \\
\hline Mouse 1 & 6.53 & 13.80 & 21.96 & 13.20 \\
Mouse 2 & 7.09 & 12.29 & 24.72 & 9.70 \\
Mouse 3 & 3.33 & 4.54 & 9.67 & 5.04 \\
Mouse 4 & 4.55 & 14.29 & 23.74 & 13.17 \\
Mouse 5 & 5.14 & 11.30 & 17.14 & 12.52 \\
Mean & $5.33 \pm 1.51$ & $11.25 \pm 1.51$ & $19.45 \pm 6.19$ & $10.73 \pm 3.49$ \\
\hline
\end{tabular}

the better delineation of the trabecular structures and in general the better contrast enable more accurate assessment of ventricular volumes and wall thickening $[22,23]$.

Cardiac MRI (CMR) in small animals was known as a cumbersome procedure suffering from low SNR, high respiratory and cardiac rates, distorted ECG signals by the magnetohydrodynamic effect, and currents introduced by the rapid gradient switching making the required cardiac synchronization difficult. With the advent of ultra-high-field MR systems SNR and hence spatial and temporal resolution of the CMR substantially increased over the last decade enabling high-quality assessment of cardiac function [24]. With the recently introduced and now widely available selfgating cardiac imaging techniques [8] CMR in small animals becomes more readily available and is increasingly applied for longitudinal studies of cardiac function. Some data had to be excluded due to motion of the animal during data acquisition. Even though not done during this study for keeping the protocol consistent, the acquisition of single corrupted slices can easily be repeated for obtaining sufficient image quality in all animals.

With the increasing use of CMR, knowledge of its reproducibility is gaining importance. Reproducibility studies of murine CMR have proved excellent intra- and interobserver variability, and correlation of CMR-derived left ventricular mass with postmortem measurements from ECGsynchronized acquisitions [24, 25]. Recently, Vanhoutte et al. reported high intra- and interobserver reproducibility and low interexperiment variability based on two subsequently performed measurements within $24 \mathrm{~h}$ with recent self-gating CMR protocols [9].

The goal of the presented study was to investigate the long-term reproducibility of cardiac function parameters derived from self-gated CMR. With the suggested imaging protocol, the interexperiment, intragroup, and longitudinal reproducibility could be quantified. For all measurement, scanning was performed according to a standardized protocol including animal positioning, scan orientation planning, and data evaluation. From the initial 6 measurements (PND 91/93/95, two measurements each day) excellent reproducibility was obtained for EF and LVM, very good for the EDV and SV, and moderate reproducibility for the ESV. In all cases no significant differences were found between the six measurements proving rather high interexperiment reproducibility. The observed values agree well with Vanhoutte et al. showing slightly lower variability for LVM, SV, and EF. Intragroup variability was assessed independently for each time point. With mean values between 7.38 (dEF) and 24.12 (dESV) the resulting intragroup variability was similar to the interexperiment variability. Long-term assessment of the variability (PND 91-PND 451) revealed highly reproducible 
cardiac function parameters over a one-year period and sensitive identification of even small changes as shown for the LVM, where a significant increase over time could be identified, while all function parameters remained unchanged.

From the data presented it is obvious that the EDV and the LVM can be quantified with highest fidelity and resulting interexperiment variability is excellent. ESV resulted as most critical parameter but still showed moderate $(<25 \%)$ variability. This may be caused by several reasons. As the temporal resolution is limited and the cardiac cycle was only resolved with 20 cardiac phases, the rather high variability of the heart rate introduced different accuracy in defining the end-systolic phase. Furthermore, due to flow artifacts within the LV cavity and the smaller end-systolic volumes, errors introduced by partial volumes effect or erroneous contoured endocardial borders especially in the most apical and basal slices introduce a larger absolute error in the derived volumes as for the EDV.

The resulting low interexperiment and intergroup variability indicate that, by using a standardized animal positioning, scan orientation planning, and data analysis approach, excellent reproducibility can be achieved in murine CMR even for long-term studies.

\section{Conclusions}

In conclusion, considering the good inter- and intraobserver reproducibility reported by other groups [9] and the excellent long-term reproducibility shown in this study, murine CMR appears as an excellent tool for longitudinal monitoring of disease progression in cardiac diseases.

\section{Abbreviations}

2CH: Two-chamber

4CH: Four-chamber

ANOVA: Analysis of variance

bpm: Beats per minute

cpm: Cycles per minute

CINE: As in cinema

CMR: Cardiovascular magnetic resonance

CoV: Coefficient of variance

ECG: Electrocardiography

EDV: End-diastolic volume

EF: $\quad$ Ejection fraction

ESV: $\quad$ End-systolic volume

FID: $\quad$ Free induction decay

FLASH: Fast Low Angle Shot

LV: $\quad$ Left ventricle

LVM: Left ventricle mass

MR: $\quad$ Magnetic resonance

MRI: Magnetic resonance imaging

MS: $\quad$ Multistack

PND: Postnatal day

SAX: $\quad$ Short axes orientations

$s_{D}: \quad$ Slice thickness

SD: $\quad$ Standard deviation

SNR: Signal-to-noise ratio

SV: $\quad$ Stroke volume
$T_{\text {ACQ }}$ : Total acquisition time

TE: Echo time

TR: Repetition time.

\section{Competing Interests}

The authors declare that they have no conflict of interests.

\section{Authors' Contributions}

Zhi Zuo was responsible for CMR image analysis, statistical analysis, and paper writing. Anne Subgang, Alireza Abaei, Wolfgang Rottbauer, and Detlef Stiller were responsible for CMR image acquisition and CMR image analysis. Genshan $\mathrm{Ma}$ was responsible for study design. Volker Rasche was responsible for study design and supervision and paper writing and modification. All authors read and approved the final manuscript. Zhi Zuo and Anne Subgang equally contributed to this work.

\section{Acknowledgments}

This work was partly funded by a research grant from the Boehringer Ingelheim Ulm University BioCenter (BIU). Grant number is PO8.

\section{References}

[1] C. Zaragoza, C. Gomez-Guerrero, J. L. Martin-Ventura et al., "Animal models of cardiovascular diseases," Journal of Biomedicine and Biotechnology, vol. 2011, Article ID 497841, 13 pages, 2011.

[2] S. D. Solomon, N. Anavekar, H. Skali et al., "Influence of ejection fraction on cardiovascular outcomes in a broad spectrum of heart failure patients," Circulation, vol. 112, no. 24, pp. 3738$3744,2005$.

[3] J. M. Gardin, F. M. Siri, R. N. Kitsis, J. G. Edwards, and L. A. Leinwand, "Echocardiographic assessment of left ventricular mass and systolic function in mice," Circulation Research, vol. 76, no. 5, pp. 907-914, 1995.

[4] D. J. Pennell, U. P. Sechtem, C. B. Higgins et al., "Clinical indications for cardiovascular magnetic resonance (CMR): consensus Panel report," European Heart Journal, vol. 25, no. 21, pp. 1940-1965, 2004.

[5] M. Dewey, M. Müller, S. Eddicks et al., "Evaluation of global and regional left ventricular function with 16-slice computed tomography, biplane cineventriculography, and two-dimensional transthoracic echocardiography. comparison with magnetic resonance imaging," Journal of the American College of Cardiology, vol. 48, no. 10, pp. 2034-2044, 2006.

[6] S. E. Slawson, B. B. Roman, D. S. Williams, and A. P. Koretsky, "Cardiac MRI of the normal and hypertrophied mouse heart," Magnetic Resonance in Medicine, vol. 39, no. 6, pp. 980-987, 1998.

[7] F. Wiesmann, J. Ruff, K.-H. Hiller, E. Rommel, A. Haase, and S. Neubauer, "Developmental changes of cardiac function and mass assessed with MRI in neonatal, juvenile, and adult mice," American Journal of Physiology-Heart and Circulatory Physiology, vol. 278, no. 2, pp. H652-H657, 2000. 
[8] B. Hiba, N. Richard, M. Janier, and P. Croisille, "Cardiac and respiratory double self-gated cine MRI in the mouse at 7 T," Magnetic Resonance in Medicine, vol. 55, no. 3, pp. 506-513, 2006.

[9] L. Vanhoutte, B. Gallez, O. Feron et al., "Variability of mouse left ventricular function assessment by 11.7 tesla MRI," Journal of Cardiovascular Translational Research, vol. 8, no. 6, pp. 362371, 2015.

[10] J. E. Schneider, K. J. Hulbert, C. A. Lygate et al., "Longterm stability of cardiac function in normal and chronically failing mouse hearts in a vertical-bore MR system," Magnetic Resonance Materials in Physics, Biology and Medicine, vol. 17, no. 3, pp. 162-169, 2004.

[11] D. Tyler, C. Lygate, J. Schneider, P. Cassidy, S. Neubauer, and K. Clarke, "CINE-MR imaging of the normal and infarcted rat heart using an $11.7 \mathrm{~T}$ vertical bore MR system," Journal of Cardiovascular Magnetic Resonance, vol. 8, no. 2, pp. 327-333, 2006.

[12] D. J. Stuckey, C. A. Carr, D. J. Tyler, and K. Clarke, "Cine-MRI versus two-dimensional echocardiography to measure in vivo left ventricular function in rat heart," NMR in Biomedicine, vol. 21, no. 7, pp. 765-772, 2008.

[13] J. Riegler, K. K. Cheung, Y. F. Man, J. O. Cleary, A. N. Price, and M. F. Lythgoe, "Comparison of segmentation methods for MRI measurement of cardiac function in rats," Journal of Magnetic Resonance Imaging, vol. 32, no. 4, pp. 869-877, 2010.

[14] Y. Wu and E. X. Wu, "MR study of postnatal development of myocardial structure and left ventricular function," Journal of Magnetic Resonance Imaging, vol. 30, no. 1, pp. 47-53, 2009.

[15] R. Zhou, S. Pickup, J. D. Glickson, C. H. Scott, and V. A. Ferrari, "Assessment of global and regional myocardial function in the mouse using cine and tagged MRI," Magnetic Resonance in Medicine, vol. 49, no. 4, pp. 760-764, 2003.

[16] A. J. Ross, Z. Yang, S. S. Berr et al., "Serial MRI evaluation of cardiac structure and function in mice after reperfused myocardial infarction," Magnetic Resonance in Medicine, vol. 47, no. 6, pp. 1158-1168, 2002.

[17] Z. Yang, C. M. Bove, B. A. French et al., "Angiotensin II type 2 receptor overexpression preserves left ventricular function after myocardial infarction," Circulation, vol. 106, no. 1, pp. 106-111, 2002.

[18] E. Heijman, J.-P. Aben, C. Penners et al., "Evaluation of manual and automatic segmentation of the mouse heart from CINE MR images," Journal of Magnetic Resonance Imaging, vol. 27, no. 1, pp. 86-93, 2008.

[19] P. Fries, A. Maßmann, R. Seidel et al., "Comparison of retrospectively self-gated and prospectively triggered FLASH sequences for cine imaging of the aorta in mice at 9.4 Tesla," Investigative Radiology, vol. 47, no. 4, pp. 259-266, 2012.

[20] E. Heiberg, J. Sjögren, M. Ugander, M. Carlsson, H. Engblom, and H. Arheden, "Design and validation of segment-freely available software for cardiovascular image analysis," BMC Medical Imaging, vol. 10, article 1, 2010.

[21] R. Ram, D. M. Mickelsen, C. Theodoropoulos, and B. C. Blaxall, "New approaches in small animal echocardiography: imaging the sounds of silence," American Journal of Physiology-Heart and Circulatory Physiology, vol. 301, no. 5, pp. H1765-H1780, 2011.

[22] A. Ghanem, W. Röll, T. Hashemi et al., "Echocardiographic assessment of left ventricular mass in neonatal and adult mice: accuracy of different echocardiographic methods," Echocardiography, vol. 23, no. 10, pp. 900-907, 2006.
[23] B. H. Amundsen, M. Ericsson, J. G. Seland, T. Pavlin, Ø. Ellingsen, and C. Brekken, "A comparison of retrospectively self-gated magnetic resonance imaging and high-frequency echocardiography for characterization of left ventricular function in mice," Laboratory Animals, vol. 45, no. 1, pp. 31-37, 2011.

[24] J. E. Schneider, P. J. Cassidy, C. Lygate et al., "Fast, high-resolution in vivo cine magnetic resonance imaging in normal and failing mouse hearts on a vertical $11.7 \mathrm{~T}$ system," Journal of Magnetic Resonance Imaging, vol. 18, no. 6, pp. 691-701, 2003.

[25] J. Ruff, F. Wiesmann, K.-H. Hiller et al., "Magnetic resonance microimaging for noninvasive quantification of myocardial function and mass in the mouse," Magnetic Resonance in Medicine, vol. 40, no. 1, pp. 43-48, 1998. 


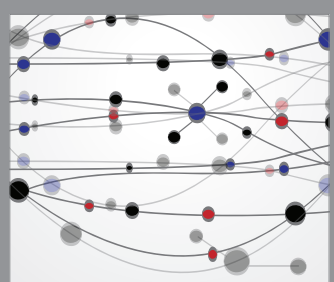

The Scientific World Journal
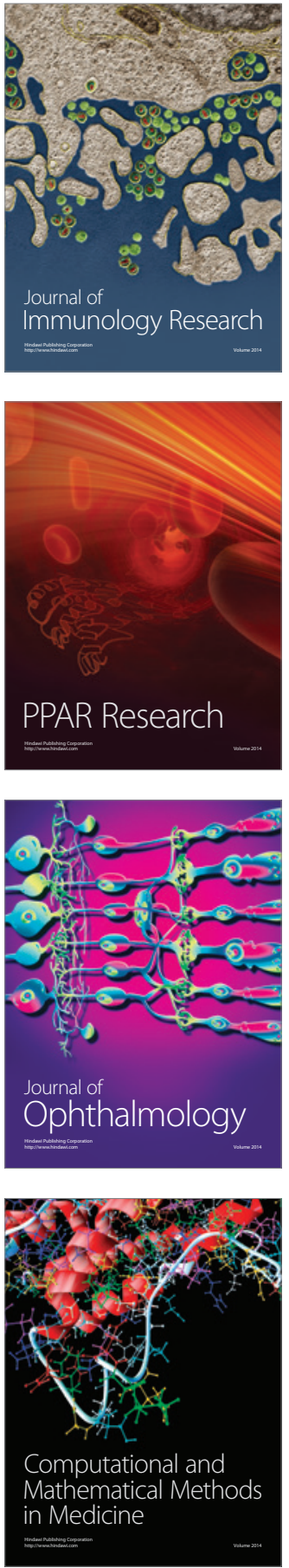

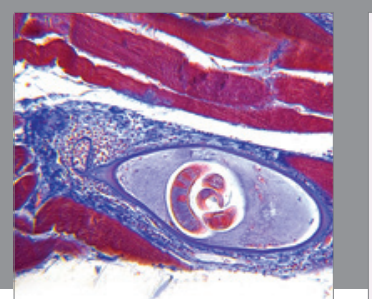

Gastroenterology Research and Practice
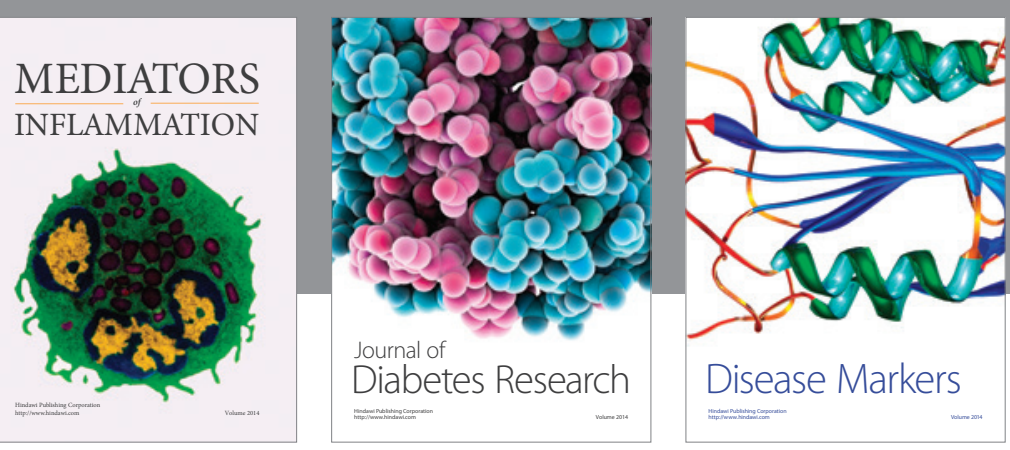

Disease Markers

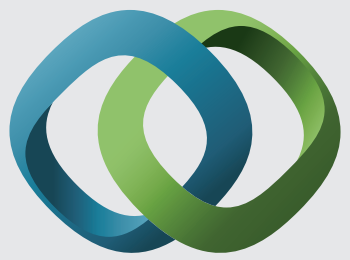

\section{Hindawi}

Submit your manuscripts at

https://www.hindawi.com
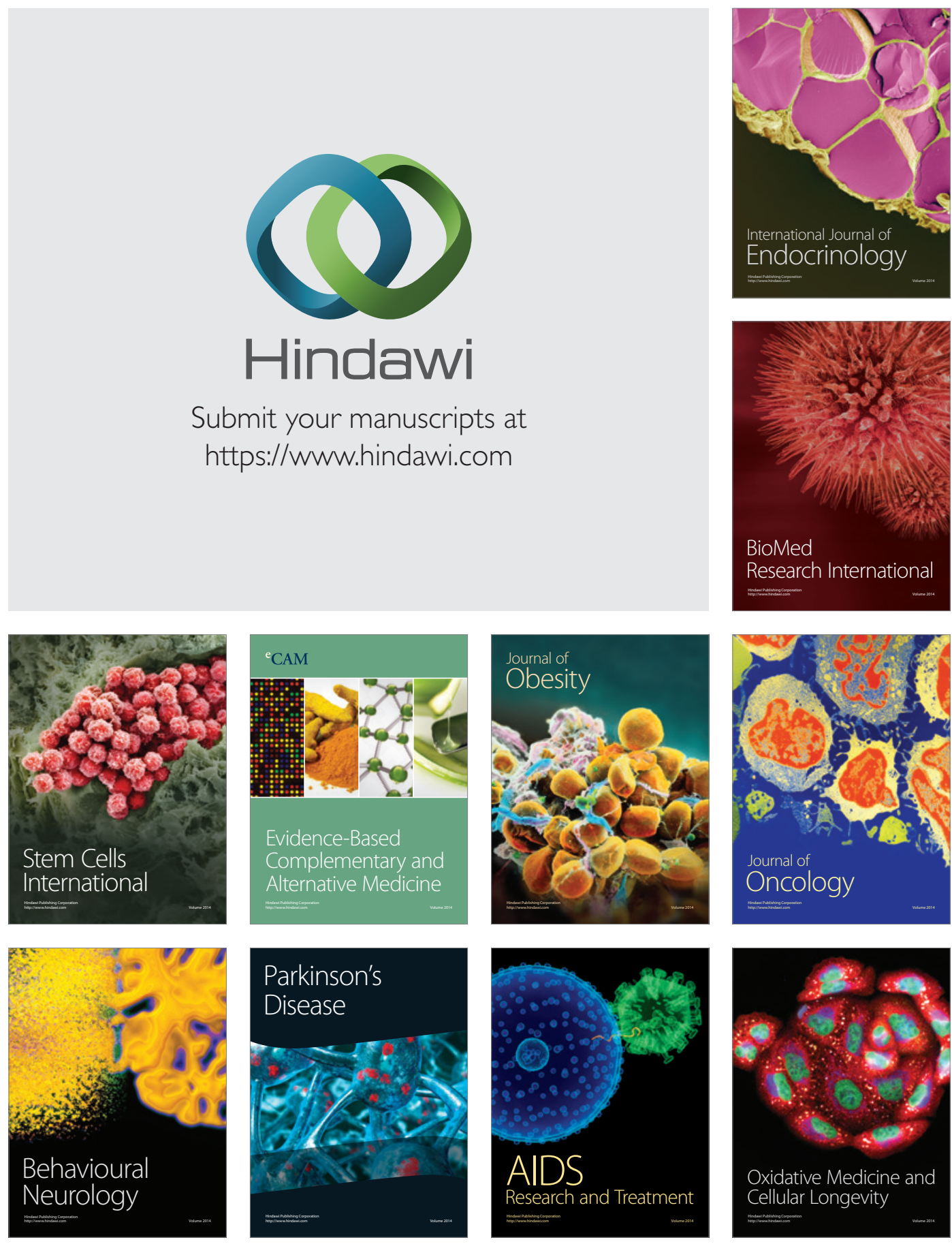